\title{
Familial juvenile hyperuricemic nephropathy type 1
}

INSERM

\section{Source}

INSERM. (1999). Orphanet: an online rare disease and orphan drug data base. Familial juvenile hyperuricemic nephropathy type 1. ORPHA:209886

Familial juvenile hyperuricemic nephropathy type $1(\mathrm{FJHN} 1)$ is a rare kidney disorder characterized by hyperuricemia, progressive nephropathy, and gout occurring at an early age. 